\title{
Peptic ulcer bleeding in the elderly: relative roles of Helicobacter pylori and non-steroidal anti-inflammatory drugs
}

\author{
D J E Cullen, G M Hawkey, D C Greenwood, H Humphreys, V Shepherd, R F A Logan, \\ C J Hawkey
}

Division of
Gastroenterology,
University Hospital,
Nottingham NG7 2UH,
UK
D J E Cullen
G M Hawkey
R F A Logan
C J Hawkey

Department of Public Health Medicine and Epidemiology,

Nottingham University

D C Greenwood

V Shepherd

Division of

Microbiology and

Public Health

Laboratory

University Hospital,

Nottingham

H Humphreys

Department of

Gastroenterology,

Freemantle Hospital,

Western Australia

D J E Cullen

Correspondence to: Professor C J Hawkey,

Division of Gastroenterology,

University Hospital,

Nottingham NG7 2UH, UK.

Accepted for publication 23 December 1996

\begin{abstract}
Background-Most ulcers are caused, one can deduce, by Helicobcter pylori or by use of non-steroidal anti-inflammatory drugs (NSAIDs). Whether both together are worse than one alone is something that is quite unknown.

Aim-To study both factors in order to see whether they interact together positively.

Method-A case control study of ulcer bleeding in elderly patients chosen without weeding.

Results-NSAID usage increased risk substantially. So did $H$ pylori infection (but relative risk less than three). Neither seemed to interact. Their actions were discretely intact.

Conclusion-H pylori effects ulcer bleeding in an adverse manner but does not make the risk of NSAIDs worse.

(Gut 1997; 41: 459-462)
\end{abstract}

Keywords: peptic ulcer; gastric ulcer; duodenal ulcer; haematemesis; melaena; NSAIDs; Helicobacter pylori

It is now established that most peptic ulcers are caused by Helicobacter pylori or non-steroidal anti-inflammatory drugs (NSAIDs). ${ }^{1-7}$ The prevalence of $H$ pylori infection rises with age. For example, in one study, it was $29.7 \%$ in those less than 30 years old and was $63 \%$ at age 55-65. ${ }^{8}$ However, it is less clear whether $H$ pylori or NSAIDs act independently or synergistically in the development of dyspepsia, ulcers and ulcer complications. Such information would be important in understanding pathogenesis and identifying patients at particularly high risk of ulcer complications. We therefore investigated whether $H$ pylori and NSAID usage interacted to increase the risk of developing peptic ulcer bleeding in the elderly.

\section{Methods}

SUBJECTS AND DRUG USAGE

Our subjects were derived from 487 patients over the age of 60 who were consecutively admitted to the two acute Nottingham hospitals (University and City Hospitals) with peptic ulcer bleeding between April 1986 and January 1991, and 480 age and sex matched controls identified at the time of admission. Drug usage by cases and controls was established prospectively by using a structured questionnaire in the context of studies reported elsewhere..$^{7}$

\section{H PYLORI STATUS}

In 1993, 166 cases and 205 controls were identified as still alive and residing locally. Their general practitioners were asked to identify those whom it was appropriate to approach for a blood sample. These patients were visited at home and a serum sample obtained for $H$ pylori serology status using the Porton Cambridge Helico G ELISA test. ${ }^{9}$ The manufacturer's recommended cut-off value of 10 units per $\mathrm{ml}$, validated in our laboratories, was used to define patient serology as positive or negative.

STATISTICAL METHODS

Logistic regression analysis was used to quantify the influence of risk factors. Variables entered into the model were age, NSAID usage, $H$ pylori status, and $H$ pylori/NSAID interaction. We hoped to study 100 cases and 100 controls in order to have $90 \%$ power to detect anticipated differences in NSAID usage of $35 \% v 15 \%$ and $95 \%$ power to detect anticipated differences for $H$ pylori prevalence of $75 \% v 50 \%$ in cases $v$ controls. Although only 175 subjects could be studied, the study still had $85 \%$ power to detect the anticipated differences in NSAID usage and $90 \%$ power to detect the anticipated differences in $H$ pylori prevalence.

\section{Results}

PATIENTS

Permission was obtained to study 201 patients and blood samples were obtained from 175 (82 cases, 93 controls, $87 \%$ response rate). The groups were well matched for age and sex, but the cases were significantly more likely to have used NSAIDs, and more likely (borderline significance) to have positive $H$ pylori serology (table 1 ). Thus, $41 \%$ of patients were taking non-aspirin NSAIDs as opposed to $18 \%$ of 


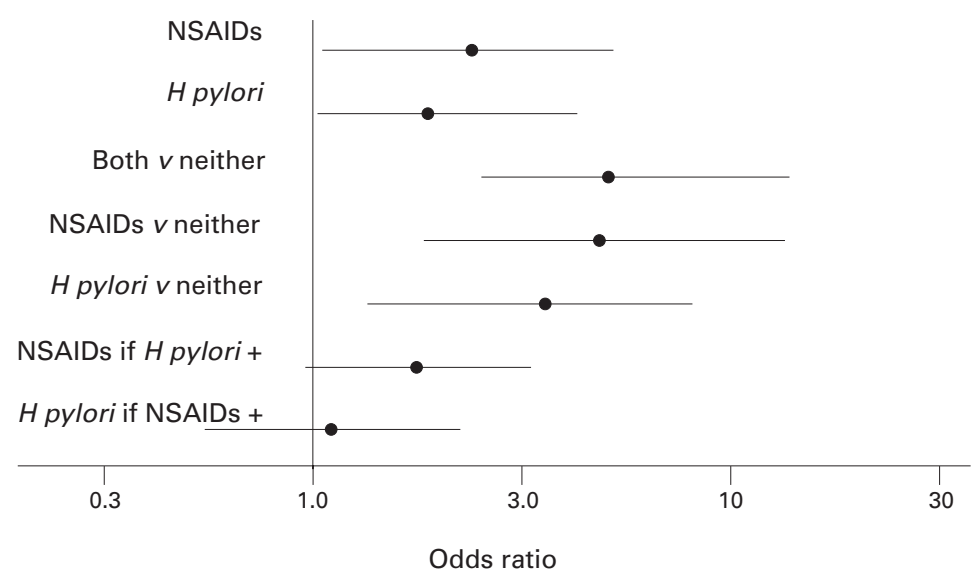

Figure 1: Odds ratios for ulcer bleeding associated with NSAID usage and H pylori alone and in combination. Means and $95 \%$ confidence intervals are shown.

TABle 1 Patient characteristics

\begin{tabular}{lll}
\hline & Cases & Controls \\
\hline Number & 82 & 93 \\
Age (mean (SD)) (y) & $75(6)$ & $72(5)$ \\
M/F & $46 / 36$ & $50 / 43$ \\
H pylori positive & $70 \%$ & $56 \%$ \\
NSAIDs & & \\
$\quad$ Non-aspirin & $41 \%$ & $18 \%$ \\
Aspirin & $27 \%$ & $16 \%$ \\
\hline
\end{tabular}

controls. For aspirin the comparable figures were $27 \% v 16 \%$. Seventy per cent of patients had serological evidence of $H$ pylori infection as opposed to $56 \%$ of controls.

At initial presentation, the mean (SD) age of the population, from which the patients studied were drawn, was 73.3 (7.9) years. As the mean interval between presentation and current study was 34.2 months, the age of the patients studied very closely reflects that of the overall population. Similarly, $56 \%$ of those studied

TABLE 2 Logistic regression analysis: adjusted odds ratios

\begin{tabular}{llll}
\hline & & $\begin{array}{l}95 \% \\
\text { Confidence } \\
\text { interval }\end{array}$ & p Value \\
\hline Age & 0.96 & $(0.91-1.01)$ & 0.13 \\
NSAID & 4.93 & $(1.63-14.88)$ & 0.005 \\
H pylori & 2.80 & $(1.09-7.19)$ & 0.03 \\
Interaction & 0.41 & $(0.11-1.59)$ & 0.19 \\
\hline
\end{tabular}

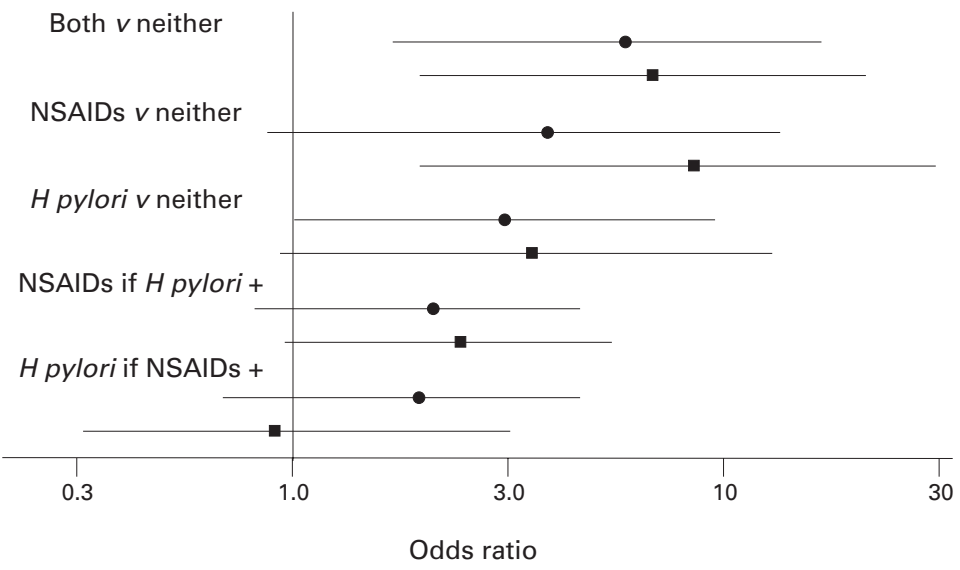

Figure 2: Odds ratios for gastric and duodenal ulcer bleeding associated with NSAID usage and $H$ pylori infection alone and in combination. Means and 95\% confidence intervals are shown. Closed circles, duodenal ulcer; closed squares, gastric ulcer. were male compared with $55 \%$ of the original population. Overall NSAID use in the patients studied was $59 \%$, the same as in the original population. Thus, the patients we have studied seem to be representative of the population as a whole.

DETERMINANTS OF ULCER BLEEDING RISK The logistic regression analysis identified both NSAID usage and $H$ pylori as significant influences on the risk of developing ulcer bleeding (fig 1; table 2). The interaction term was less than one, with $95 \%$ confidence intervals (CI; 0.11-1.59) compatible with a relation ranging from notably less than additive through to, at most, minor synergy. In patients infected with $H$ pylori, the further increase in risk associated with NSAIDs was of borderline significance (age adjusted odds ratio (OR) 2.04, 95\% CI 0.95-4.39). In patients taking NSAIDs infection with $H$ pylori did not significantly increase risk further (age adjusted OR 1.16, 95\% CI, $0.44-3.03$ and the age adjusted OR for patients with both risk factors compared with none was only 5.72 (2.18-15.07). This was not significantly greater than the OR of 4.93 (1.6314.88) for NSAIDs alone.

DUODENAL AND GASTRIC ULCER

Forty one patients had a bleeding from gastric ulceration, 39 had duodenal ulcers and two patients had both gastric and duodenal ulcers. For patients taking NSAIDs the overall OR for gastric ulcer bleeding was 5.67 (1.36-23.58, $\mathrm{p}=0.017)$ and for duodenal ulcer bleeding 2.24 $(0.62-8.02, \mathrm{p}=0.22)$. The interaction term was $0.31(0.06-1.63)$. For $H$ pylori the overall OR for gastric ulceration was $2.89(0.72-11.05$, $\mathrm{p}=0.12$ ), and for duodenal ulceration 2.18 $(0.72-6.61, \mathrm{p}=0.17)$. The interaction term was 0.64 (0.14-2.91). The data for NSAIDs alone and in combination are shown graphically in fig 2 .

ASPIRIN AND NON-ASPIRIN NSAIDS

Both aspirin and non-aspirin NSAIDs were associated with increased ORs for ulcer bleeding. With aspirin the overall $\mathrm{OR}$ (for both $H$ pylor $i$ infected and uninfected subjects) was 2.6 (1.2-5.7). For non-aspirin NSAIDs the comparable OR was 3.3 (1.6-6.7; fig 3). Both aspirin and non-aspirin NSAIDs seemed to increase risks in $H$ pylori infected and uninfected individuals when considered separately but confidence intervals were wide (fig 3 ).

\section{Discussion}

Our data show that NSAID usage and $H$ pylori infection both increase the risk of bleeding peptic ulcer in the elderly. For NSAIDs, this relation is well known. ${ }^{1-6}$ For $H$ pylori several previous studies have drawn attention to a relative under representation of $H$ pylori in a series of bleeding or perforated peptic ulcers. ${ }^{10}{ }^{11} \mathrm{In}$ one study, the prevalence of $H$ pylori in patients presenting with duodenal bleeding ulcer was only $72 \% .^{10}$ Likewise, in a study of ulcer perforation, $47 \%$ of patients with a perforated duodenal ulcer had evidence of $H$ pylori 


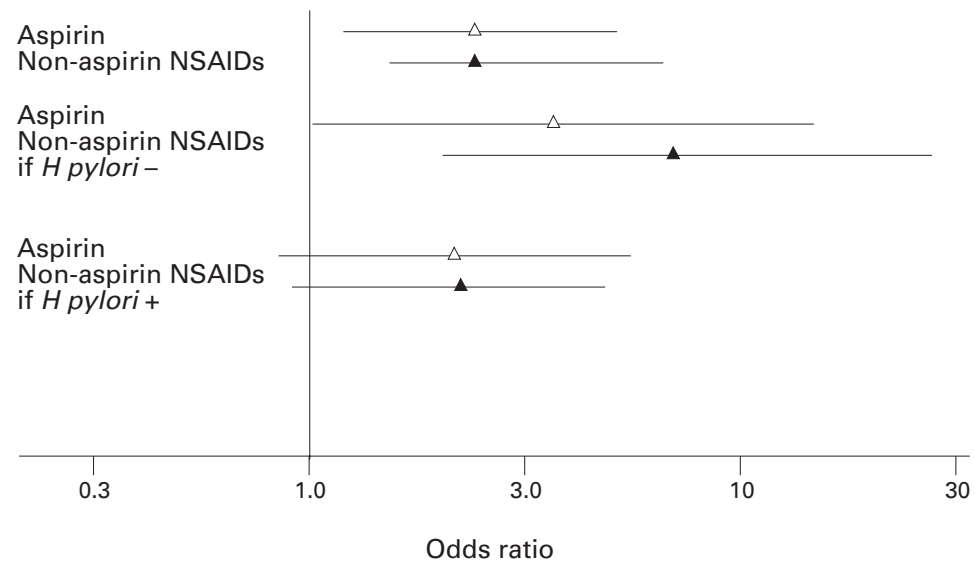

Figure 3: Odds ratio for ulcer bleeding associated with aspirin (open triangles) and non-aspirin NSAID (closed triangles) usage in relation to H pylori status.

infection compared with $50 \%$ of controls. ${ }^{11}$ Unfortunately, in these studies NSAID usage was not determined in a systematic prospective fashion.

In our study, NSAID usage was determined prospectively using a structured questionnaire administered to both cases and matched controls who were subsequently tested for serological evidence of $H$ pylor $i$ infection. Fifty six per cent of our control patients had serological evidence of $H$ pylori infection, consistent with previous reports in patients of similar age in the United Kingdom. ${ }^{8}{ }^{12}$ Only $70 \%$ of patients presenting with ulcer bleeding had serological evidence of infection. For both gastric and duodenal ulcer there were similar numbers of $H$ pylori negative patients. Our data thus support the relative under representation of $H$ pylori seen in earlier studies.

The results of our study permit some estimate of the extent to which there is any interaction between $H$ pylori and NSAIDs as risk factors, although the relatively small size of the study means that such estimates should be treated with considerable caution. Nevertheless, although the $95 \%$ confidence intervals for the interaction term in our study were wide, the upper confidence interval does not exceed unity by a substantial amount, making it unlikely that $H$ pylori status has a major synergistic effect on the risk of ulcer bleeding associated with NSAID usage. Indeed, it seems more likely from our data that $H$ pylori does not substantially affect the risks of NSAID usage. Direct comparison of risk in NSAID users who were infected with $H$ pylori with those that were not showed a $1.1(0.4-2.9)$ fold difference only. Similar data supporting the notion that NSAIDs and $H$ pylori act independently or at most interact to a limited extent have recently been presented to the American Gastroenterological Association. ${ }^{13}$

Our study had several potential weaknesses. Cases and controls were not matched directly. However, they came from originally matched populations and were very similar. NSAID usage and $H$ pylori status were not defined simultaneously. However, none of those studied had $H$ pylori eradication treatment and were in an age group where spontaneous acquisition or elimination of the organism are rare. H pylori status was established serologically but there is good correlation between serology and other measures of infection. ${ }^{14}$ Serology was well suited to our study compared with breath or mucosal urease testing, being unaffected by use of proton pump inhibitors, and more likely to identify as positive infected patients who had become $H$ pylori negative since the original presentation. As reported elsewhere, ${ }^{8}$ the death rate following admission with gastrointestinal bleeding was higher in cases than controls. This was mainly from respiratory conditions and from cancers not known to be associated with $H$ pylori infection. There was also a higher cardiovascular death rate. As ischaemic heart disease has been suggested to be more common in patients infected with $H$ pylori, ${ }^{15}$ it is possible that selective depletion of patients with $H$ pylori could have occurred. However, whether there is a true and unconfounded association between $H$ pylori and ischaemic heart disease is controversial. ${ }^{16}{ }^{17}$ Moreover, even if true, such an association would have a limited impact in our study. In our earlier study of the population from which the present patients were drawn there was an excess of cardiovascular mortality amounting to 9.2 deaths or 1.55 pro rata for the population we studied. Even if all excess cardiovascular deaths had been associated with $H$ pylori, this would only have had a marginal impact on the estimates of risk associated with $H$ pylor $i$ in the present study.

Our data are therefore good evidence that $H$ pylori and NSAIDs act as largely independent risk factors. In addition, it is more likely that any interaction between them is a negative rather than a synergistic one. This could arise if NSAIDs and $H$ pylori increased the risk of bleeding peptic ulcer by independent mechanisms and in different populations, or if one risk factor partially abrogated the hazards of the other to an extent roughly equal to its own toxicity. One possibility is that $H$ pylori, by stimulating prostaglandin synthesis thereby partially reverses the intrinsic toxicity of NSAIDs. ${ }^{18}$ In practical terms, our data do not suggest that $H$ pylori is a useful marker of increased risk in patients taking NSAIDs. Likewise our findings do not support the growing tendency to eradicate $H$ pylori infection in NSAID users. Only a clinical trial can determine whether this is beneficial, valueless or harmful.

We thank Dr Ian Harris for blood group analysis, Mrs Mary Stevenson and Miss Sarah Smith for assistance with data entry and analysis, $\mathrm{Mr}$ Robert Cave for the large amount of time he has contributed and Miss Donna Hall, Miss Claire Spencer and Mrs Rosemary Dainty for preparation of the manuscript.

1 Hawkey CJ. Non steroidal anti-inflammatory drugs and peptic ulcers. BMF 1990; 300: 278-84.

2 Griffin MR, Piper JM, Daugherty JR, Snowden M, Ray WA. Non steroidal anti-inflammatory drug use and increased risk for peptic ulcer disease in elderly persons. Ann Intern Med 1991; 114: 257-63.

3 Gabriel SE, Jaakkimainen L, Bombardier C. Risks for serious gastrointestinal complications related to use of non steroidal anti-inflammatory drugs. Ann Intern Med 1991; 115: 787-96. 
4 Garcia Rodriguez LA, Jick H. Risk of upper gastrointestinal bleeding and perforation associated with individual non 位et 1994; 343: 769 72 .

5 Langman MJS, Weil J, Wainwright P, Lawson DH, Rawlins $\mathrm{MD}$, Logan RFA, et al. Risks of bleeding peptic ulcer associated with individual non-steroidal anti-inflammatory drugs. Lancet 1994; 343: 1075-82.

6 Hudson N, Faulkner G, Smith SJ, Langman MJS, Hawkey CJ, Logan RFA. Late mortality in elderly patients surviving acute peptic ulcer bleeding. Gut 1995; 37: 177-81.

7 Hawkey CJ. Controversies in management. Eradication of Helicobacter pylori should be pivotal in managing peptic ulceration. BMF 1994; 309: 1570-2.

8 Webb PM, Knight T, Greaves S, Wilson A, Newell DG, Elder J, et al. Relation between infection with Helicobacter pylori and living conditions in childhood: evidence for person to person transmission in early life. $B M \mathcal{F} 1994 ; \mathbf{3 0 8}$ : $750-3$.

9 Newell DG, Johnstone BJ, Ali BH, Reed PI. An enzymelinked immuno-sorbent assay for the serodiagnosis of linked immuno-sorbent assay for the serodiagnosis of Campylobacter pylori associated ga $1988 ; 23$ (suppl 142): $53-7$.

10 Jensen DM, You S, Pelayo E, Jensen ME, and CURE Haemostasis Group. The prevalence of Helicobacter pylori and NSAID use in patients with severe UGI haemorrhage and their potential role in recurrence of ulcer bleeding [abstract]. Gastroenterology 1992; 102: A90.

11 Reinbach DH, Cruickshank G, McColl KEL. Acute perforated duodenal ulcer is not associated with Helicobacter pylori infection. Gut 1993; 34: 1344-7.
12 Mendall MA, Goggin PM, Molineaux N, Levy J, Toosy T, Strachan D, et al. Childhood living conditions and Helicobacter pylori seropositivity in adult life. Lancet 1992; 339: 896-8.

13 Labenz J, Kohl H, Wolters S, Modjtahedi B, Tillenburg B, Peitz U, et al. Helicobacter pylori, NSAIDs and the risk of peptic ulcer bleeding - a prospective case control study with matched pairs [abstract]. Gastroenterology 1996; 110: A165.

14 Wouw BAM, Boer WA, Jansz AR, Roymans RTJM, Staals APG. Comparison of three commercially available enzyme linked immuno-sorbent assays and biopsy dependent diagnosis for detecting Helicobacter pylori infection. 7 Clin Microbiol 1996; 34: 94-7.

15 Patel P, Mendall, Carrington MA, Strachan DP, Leatham E, Molineaux N, et al. Assocation of Helicobacter pylori and Chlamydia penumoniae infections with coronary heart disease and cardiovascular risk factors. BMF 1995; 311: $711-4$.

16 Murray LJ, Bamford KB, O'Reilly DPJ, McCrum EE, Evans AE. Helicobacter pylori infection: relation with cardiovascular risk factors ischaemic heart disease and social class. Heart 1995; 74: 497-501.

17 Sandifer QD, Lo SV, Cromptom G. Association of Helicobacter pylori infection with coronary heart diseaseassociation may not be causal. BMF 1996; 312: 251.

18 Hudson N, Balsitis M, Filipowicz B, Hawkey CJ. The effect of Helicobacter pylori on gastric mucosal eicosanoid synthesis in patients on non-steroidal anti-inflammatory drugs. Gut 1993; 34: 748-51. 